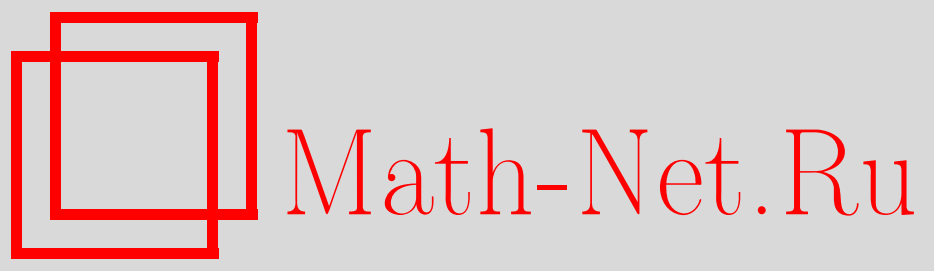

А. Э. Хузиева, Д. А. Шабанов, Об однородных гиперграфах с большим обхватом и большим хроматическим числом, Дискрет. матем., 2015, том 27, выпуск 2, 112133

DOI: https://doi.org/10.4213/dm1329

Использование Общероссийского математического портала Math-Net.Ru подразумевает, что вы прочитали и согласны с пользовательским соглашением http: //www . mathnet.ru/rus/agreement

Параметры загрузки:

IP: 52.87 .193 .239

26 апреля 2023 г., 13:19:38

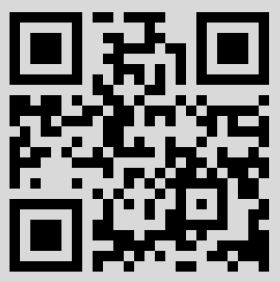




\title{
Об однородных гиперграфах с большим обхватом и большим хроматическим числом
}

\author{
() 2015 г. А. Э. Хузиева*, Д. А. Шабанов**
}

В работе исследуется экстремальная задача комбинаторного анализа об отыскании минимально возможного количества ребер в $n$-однородном гиперграфе с хроматическим числом больше $r$ и обхватом больше $s$. Получена новая нижняя оценка подобной экстремальной величины, а также ряд смежных результатов.

Работа выполнена при поддержке Программы поддержки ведущих научных школ (грант № НШ-2964.2014.1) и гранта Президента РФ МК-692.2014.1.

Ключевые слова: гиперграф, раскраски гиперграфов, разреженные гиперграфы, метод случайной перекраски, обхват гиперграфа.

\section{1. Введение и история задачи}

Работа посвящена изучению известной проблемы экстремальной комбинаторики, связанной с раскрасками гиперграфов. Напомним основные определения из теории гиперграфов.

Гиперграфом $H$ называется пара множеств $H=(V, E)$, где $V=V(H)$ - это некоторое конечное множество, элементы которого называются вершинами гиперграфа, а $E=E(H)$ - произвольная совокупность подмножеств $V$, которые принято называть ребрами гиперграфа $H$. Если каждое ребро $A \in E$ состоит ровно из $n$ вершин (т.е. $A$ - это $n$-подмножество $V$ ), то говорят, что гиперграф $H$ является n-однородным.

Раскраской множества вершин гиперграфа $H=(V, E)$ называется любое отображение $f: V \rightarrow \mathbb{N}$. Раскраска $f$ является правильной для гиперграфа, если в ней нет одноцветных ребер. Формально, для каждого $A \in E$ выполнено

$$
|\{f(v): v \in A\}|>1 .
$$

Минимальное число цветов, необходимое для правильной раскраски гиперграфа $H$, называется его хроматическим числом и обозначается $\chi(H)$.

Cmепенъю вершины $v$ гиперграфа $H$ называется количество ребер $H$, содержащих $v$. Максимальную степень вершины гиперграфа $H$ мы будем обозначать через $\Delta(H)$. Циклом длины $k$ в гиперграфе $H=(V, E)$ называется чередующаяся последовательность $v_{0}, A_{1}, v_{1}, \ldots, A_{k}, v_{k}=v_{0}$ из $k$ различных вершин $v_{0}, \ldots, v_{k-1}$ и

* Место работы: Московский физико-технический институт (государственный университет), e-mail: atomochek@gmail.com

** Место работы: МГУ им. М. В. Ломоносова, e-mail: dm.shabanov.msu@gmail.com 
$k$ различных ребер $A_{1}, \ldots, A_{k}$ с условием, что для любого $i=1, \ldots, k$ выполнено $v_{i-1} \in A_{i}$ и $v_{i} \in A_{i}$. Длина минимального цикла в гиперграфе называется его обхватом. Мы будем обозначать обхват гиперграфа $H$ через $g(H)$.

Заметим, что в отличие от случая графов гиперграфы могут иметь обхват равный двум. Цикл длины два в гиперграфе возникает, если в нем найдутся два ребра, имеющих хотя бы две общие вершины. Если же $g(H)>2$, то каждые два различных ребра имеют не более одной общей вершины. Подобные гиперграфы (по аналогии с графами) принято называть простыми.

Изучение взаимосвязи значений хроматического числа, обхвата и максимальной степени вершины у $n$-однородных гиперграфов началось с классической работы П. Эрдеша и Л. Ловаса [1] 1973 г. В ней доказано, что для любых $n \geqslant 3, r \geqslant 2$, $s \geqslant 2$ существует $n$-однородный гиперграф $H$ с хроматическим числом больше $r$ и обхватом больше $s$. Более того, они показали, что можно ограничить сверху число ребер и максимальную степень вершины в таком гиперграфе.

Теорема 1 (П. Эрдеш, Л. Ловас, [1]). Пусть $n \geqslant 3, r \geqslant 2, s \geqslant 2$. Тогда существует $n$-однородный гиперграф $H$ со следующими свойствами: $\chi(H)>r, g(h)>s$,

$$
\begin{gathered}
|E(H)| \leqslant 4 \cdot 20^{s} n^{3 s-2} r^{(n+1) s}, \\
\Delta(H) \leqslant 20 n^{2} r^{n+1} .
\end{gathered}
$$

Тем самым, Эрдеш и Ловас мотивировали изучение экстремальной величины $m(n, r, s)$, определяемой как минимально возможное количество ребер гиперграба в классе п-однородных гиперграфов с хроматическим числом больше $r$ и обхватом больше s. Формально,

$$
m(n, r, s)=\min \{|E(H)|: H-n \text {-однородный, } \chi(H)>r, g(H)>s\} \text {. }
$$

При $s=1$ условие на обхват тривиально, в этом случае мы рассматриваем весь класс $n$-однородных гиперграфов с большим хроматическим числом. Величина $m(n, r, 1)$ достаточно хорошо изучена, вопрос ее нахождения - это классическая проблема Эрдеша-Хайнала, подробнее о которой можно прочитать, например, в обзоре [2].

В ситуации с нетривиальным обхватом наиболее хорошо изучен случай простых гиперграфов, т.е. величина $m(n, r, 2)$. Согласно теореме 1

$$
m(n, r, 2) \leqslant 1600 n^{4} r^{2 n+2} .
$$

Однако, как оказалось позднее, данная оценка не дает правильный порядок асимптотики ни по $n$ (при фиксированном $r$ ), ни по $r$ (при фиксированном $n$ ). В первой ситуации наилучшую верхнюю оценку получили А.В. Косточка и В. Рёдль в [3]: при всех $n>n_{0}(r)$ выполнено

$$
m(n, r, 2) \leqslant 16 e^{2 r} n^{2} r^{2 n}(\ln r)^{2} .
$$

Наилучшая из известных нижних оценок при фиксированном $r$ была обоснована в недавней работе Я. Козика и Д.А. Шабанова [4]:

$$
m(n, r, 2) \geqslant c r^{2 n-4},
$$


где $c>0-$ некоторая абсолютная константа. Отметим, что зазор между оценками (2) и (3) имеет порядок $n^{2}$ при фиксированном $r \geqslant 2$.

В ситуации, когда $n$ фиксировано, а $r$ растет, величина $m(n, r, 2)$, как показали А.В. Косточка, Д. Мубаи, В. Рёдль и П. Тетали в работе [5], имеет порядок $r^{2 n-2}(\ln r)^{2}$. Они обосновали неравенства

$$
c(n) r^{2 n-2}(\ln r)^{2} \leqslant m(n, r, 2) \leqslant 8 n^{10} r^{2 n-2}(\ln r)^{2},
$$

где функция $c(n)>0$ ими не была явно выписана (из доказательства, впрочем, следует, что $c(n)$ можно взять равной $\left.10^{-n}\right)$. По-видимому, наилучшую из известных верхних оценок в рассматриваемой ситуации можно вывести из второго результата статьи [3], в котором Косточка и Рёдль дают вероятностную конструкцию $n$-однородного гиперграфа с большим обхватом и большим хроматическим числом. В нем они уточняют оценку для максимальной степени вершины в подобном гиперграфе ( $\left\lceil n r^{n-1} \ln r\right\rceil$ вместо $20 n^{2} r^{n+1}$ в теореме 1$)$, однако явно не выписывают оценку для числа ребер получающегося гиперграфа. Но из используемой ими работы Сауэра [6] о регулярных гиперграфах с большим обхватом следует, что

$$
m(n, r, 2) \leqslant 4 n^{5} r^{2 n-2}(\ln r)^{2} .
$$

Это примерно в $n^{5}$ раз улучшает верхнюю оценку из (4).

В отличие от случая простых гиперграфов, задача для гиперграфов с более сильными ограничениями на обхват изучена намного хуже. Верхнюю оценку для $m(n, r, s)$ Эрдеша-Ловаса из теоремы 1 для произвольного $s$ можно уточнить следующим образом:

$$
m(n, r, s) \leqslant 2 e^{2} s e^{s} n^{3 s-2} r^{s(n+1)} .
$$

Вторая известная конструкция гиперграфов с большим обхватом и большим хроматическим числом - это уже упоминавшийся результат Косточки и Рёдля из [3]. Их конструкция в сочетании с теоремой Сауера [6] дает более сильную верхнюю оценку, нежели (5):

$$
m(n, r, s) \leqslant 2 s n^{2 s+1} r^{s(n-1)}(\ln r)^{s} .
$$

Легко видеть, что (6) усиливает (5) для всех значений параметров $n, r$ и $s \geqslant 3$.

Единственная же нетривиальная нижняя оценка $m(n, r, s)$ для $s>2$ была получена Косточкой и Кумбхатом в работе [7]. Они показали, что для любых $s, r \geqslant 2$, $\varepsilon>0$ найдется такое $n_{0}=n_{0}(r, s, \varepsilon)$, что при всех $n>n_{0}$

$$
\begin{gathered}
m(n, r, s)>r^{n(\lfloor s / 2\rfloor+1)} n^{1-\varepsilon} \text { при нечетном } s ; \\
m(n, r, s)>r^{n(\lfloor s / 2\rfloor+1)} n^{-\varepsilon} \text { при четном } s .
\end{gathered}
$$

Заметим, что зазор между оценками (6) и (7) имеет экспоненциальный порядок роста по $n$ при фиксированных $r \geqslant 2, s>2$. Таким образом в задаче об $m(n, r, s)$ неизвестна даже асимптотика логарифма $\ln (m(n, r, s)$,$) .$

Перейдем к формулировкам полученных в работе новых результатов. 


\section{2. Новые результаты}

В основе доказательства нижней оценки (7) для величины $m(n, r, s)$ Косточкой и Кумбхатом был использован некоторый структурный результат об устройстве простых гиперграфов. Для его точной формулировки нам понадобится ряд определений.

Пусть $H=(V, E)-n$-однородный гиперграф, а $\delta-$ некоторое положительное число. Вершина $v \in V$ называется $\delta$-легкой, если ее степень в гиперграфе $H$ не превосходит $\delta$. В противном случае, вершина называется $\delta$-тлжелой. Ребро называется $\delta$-тяжелым, если в нем более половины вершин являются $\delta$-тяжелыми. В противном случае ребро называется $\delta$-легким.

В работе Косточки и Кумбхата была доказана следующая теорема о раскрасках простых гиперграфов.

Теорема 2 (А.В. Косточка, М. Кумбхат, [7]). Для любых $\varepsilon>0$ u $r \geqslant 2$ найдется такое $n_{0}=n_{0}(\varepsilon, r)$, что для любого $n>n_{0}$ и любого $n$-однородного простого гиперграфа, удовлетворяющего условиям

1)

$$
\Delta(H) \leqslant n r^{n-1},
$$

2) каждая вершина содержится в не более чем в $r^{n-1} n^{-\varepsilon} \delta$-тяљельх ребрах с $\delta=r^{n-1} n^{-\varepsilon}$, справедлива оценка $\chi(H) \leqslant r$.

Смысл теоремы 2 состоит в том, что если гиперграф $H$ не является $r$-раскрашиваемым, то либо в нем найдется вершина уж очень большой степени, не менее $n r^{n-1}$, либо найдется вершина, в окрестности которой найдется много (порядка $\left.r^{n-1} n^{-\varepsilon}\right)$ вершин просто большой степени, не меньше $r^{n-1} n^{-\varepsilon}$.

Основным результатом настоящей работы является следующее улучшение структурного результата Косточки и Кумбхата для случая гиперграфов с обхватом больше пяти.

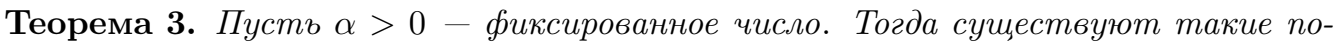
ложительные $c=c(\alpha)$ и $n_{0}=n_{0}(\alpha)$, что при $\delta=c \cdot r^{n-1}$ u $n>n_{0}$ для любого $n$-однородного гиперграфа $H=(V, E)$ с обхватом больше пяти, удовлетворяющего

$$
\Delta(H) \leqslant n^{\alpha} r^{n-1},
$$

каждая вершина $H$ содержится не более чем в $c \cdot r^{n-1} \delta$-тяжельх ребрах, справедлива оценка $\chi(H) \leqslant r$.

Количественные оценки в теореме 3 достаточно близки к оптимальным. Как уже упоминалось, Косточка и Рёдль показали (см. [3]), что существуют $n$-однородные гиперграфы с хроматическим числом больше $r$, со сколь угодно большим обхватом и максимальной степенью вершины не более $\left\lceil n r^{n-1} \ln r\right\rceil$. Значит, в формулировке теоремы величина $\delta$ не может превышать $n r^{n-1} \ln r$, что не более чем в $n \ln r$ раз превышает условие на $\delta$ в нашей теореме.

Выделим одно простое следствие из теоремы 3.

Следствие 1. Существует такое положительное число $c>0$, что для любого $n$-однородного гиперграфа $H=(V, E)$ с обхватом больше пяти и хроматическим числом больше $r$

$$
\Delta(H) \geqslant c r^{n-1} .
$$


Этот результат крайне интересен сам по себе, получением подобных оценок занималось много крупных специалистов по экстремальной комбинаторике (см., например, [2]). Однако он не является новым, а был ранее получен в работе [4] Козика и Шабанова для более широкого класса простых гиперграфов.

В качестве второго следствия мы получаем улучшение нижних оценок (7) величины $m(n, r, s)$.

Следствие 2. Существует такая абсолютная константа $c>0$, что при $5 \leqslant s<n / 2$

$$
\begin{gathered}
m(n, r, s) \geqslant c^{\lfloor s / 2\rfloor} r^{(n-s)(\lfloor s / 2\rfloor+1)} \text { если s четно; } \\
m(n, r, s) \geqslant c^{\lfloor s / 2\rfloor} n r^{(n-s)(\lfloor s / 2\rfloor+1)} \text { ecли s нечетно. }
\end{gathered}
$$

Доказательство. Доказательство следует рассуждениям Косточки и Кумбхата. Пусть $c_{0}-$ константа из формулировки теоремы 3 для $\alpha=1$. Пусть также $H=$ $(V, E)$ - такой $n$-однородный гиперграф, что $\chi(H)>r, g(H)>s$.

Пусть $x, d$ - положительные числа. Ребро гиперграфа назовем $(x, d)$-тяжелым, если в нем не менее $x$ вершин имеют степень не меньше $d$. Для вершины $v \in V$ ее $(x, d)$-степенъю называется количество $(x, d)$-тяжелых ребер, содержащих эту вершину.

Рассмотрим две операции изменения гиперграфа: если $H^{\prime}$ - гиперграф, то при фиксированных $(x, d)$

- $\mathcal{M}\left(H^{\prime}\right)$ получается из $H^{\prime}$ удалением из каждого ребра вершины максимальной степени (если есть несколько вершин максимальной степени, то выбираем любую);

- $\mathcal{D}\left(H^{\prime}\right)$ получается из $H^{\prime}$ удалением из каждого ребра вершины максимальной $(x, d)$-тяжелой степени (если есть несколько вершин максимальной $(x, d)$-тяжелой степени, то выбираем любую).

Заметим, что введённые операции удаляют вершины только из ребер, а не из гиперграфа, тем самым, множество вершин в гиперграфах $\mathcal{M}\left(H^{\prime}\right)$ и $\mathcal{D}\left(H^{\prime}\right)$ совпадает с множеством вершин $H^{\prime}$. Следовательно, обе операции переводят $k$-однородный гиперграф в $(k-1)$-однородный. Обозначим через $\mathcal{M}^{t}\left(H^{\prime}\right)\left(\mathcal{D}^{t}\left(H^{\prime}\right)\right)$ - гиперграф, получающийся последовательным применением первой (второй) операции к гиперграфу $H^{\prime}$.

Обозначим $l=\left\lfloor\frac{s}{2}\right\rfloor$, тогда $s=2 l+1$ для нечетных $s$ и $s=2 l$ в случае четного $s$. Рассмотрим следующие гиперграфы

$$
H_{1}=\mathcal{M}^{l}(H), \quad H_{2}=\mathcal{D}^{l-1}\left(H_{1}\right)=\mathcal{D}^{l-1} \mathcal{M}^{l}(H),
$$

где параметры $(x, d)$ выбраны следующим образом:

$$
x=\frac{n-2 l+1}{2}, \quad d=\delta(n-2 l+1)=c_{0} r^{n-2 l} .
$$

Легко видеть, что гиперграф $H_{2}$ будет $(n-2 l+1)$-однородным, его хроматическое число тоже будет больше $r$, а обхват - больше $s$. Тогда согласно теореме 3 возможна следующая альтернатива: либо $\Delta\left(H_{2}\right)>(n-2 l+1) r^{n-2 l}>(n / 2) r^{n-2 l}$, либо в $H$ найдется вершина $(x, d)$-тяжелой степени не меньше $\delta(n-2 l+1)$ (заметим, что для $H_{2}$ тяжелые ребра - это в точности $\delta(n-2 l+1)$-тяжелые). Рассмотрим по отдельности оба варианта. 
1) Если $\Delta\left(H_{2}\right)>\frac{1}{2} n r^{n-2 l}$, то тогда, конечно, и $\Delta\left(H_{1}\right)=m>\frac{1}{2} n r^{n-2 l}$. Далее, лемма 8 из работы [7] Косточки и Кумбхата утверждает, что в этом случае исходный гиперграф $H$ должен содержать не меньше $m^{l}$ различных вершин, каждая из которых имеет степень не меньше $m$ (достаточно восстановить ранее удаленные вершины в процессе применения оператора $\mathcal{M}$ ). Следовательно, общее число ребер в $H$ можно оценить снизу следующим образом:

$$
|E| \geqslant \frac{m \cdot m^{l}}{n}>\frac{1}{n}\left(\frac{1}{2} n r^{n-2 l}\right)^{l+1} \geqslant\left(\frac{1}{2}\right)^{\lfloor s / 2\rfloor+1} n r^{(n-s)(\lfloor s / 2\rfloor+1)} .
$$

(последний переход использует соотношения $l+1=\lfloor s / 2\rfloor+1 \geqslant 3,2 l \leqslant s$ )

2) Осталось рассмотреть второй случай, когда $H_{2}$ содержит вершину большой тяжелой степени. Здесь мы снова воспользуемся леммой Косточки и Кумбхата из [7].

Лемма 1 (А.В. Косточка, М. Кумбхат, [7]). Пусть $H^{\prime}$ - гиперграф с обхватом строго больше $2 t$, а $H^{\prime}=\mathcal{D}^{t}(H)$ с некоторыми параметрами $x$ u $d$. Если $H^{\prime}$ содержит вершину $v$ c $(x, d)$-тяжелой степенъю не менъше $m$, то $H$ содержит не менее $(m-1)^{t}$ различных вершин, $(x, d)$-тяжелая степень каждой из которьх не менъше $m$ и которые находятся на реберном расстоянии ровно $t$ от $v$.

Применим лемму 1 к гиперграфам $H_{1}$ и $H_{2}=\mathcal{D}^{l-1}\left(H_{1}\right)$. Согласно ей в $H_{1}$ найдется вершина $v$ с $(x, d)$-тяжелой степенью $m \geqslant c_{0} r^{n-2 l}$, а также $(m-1)^{l-1}$ вершин на расстоянии ровно $l-1$ от $v$ с тем же ограничением на $(x, d)$-тяжелую степень. Обозначим полученное множество вершин через $W$. Для каждой вершины $u \in W$ найдется ровно один реберный путь длины $l-1$ до исходной вершины $v$ (иначе в $H_{1}$ появился бы цикл длины не более $2 l \leqslant s)$. Обозначим через $A(u)$ последнее ребро в подобном пути. Если некоторое ребро $B(u) \neq A(u)$ также содержит $u \in W$, то оно не может содержать другие вершины на расстоянии не более $l$ от $v$, иначе мы получаем цикл длины не более $2 l \leqslant s$ в $H_{1}$. Тем самым,

$$
\begin{gathered}
B(u) \cap B\left(u^{\prime}\right)=\varnothing \text { если } u \neq u^{\prime}, u, u^{\prime} \in W, \\
B(u) \cap C(u)=\{u\} .
\end{gathered}
$$

Для каждой вершины $u \in W$ число таких $(x, d)$-тяжелых ребер не меньше $m-1$, в каждом из них есть не менее $(x-1)$-й вершины степени не меньше $d$, которые не принадлежат другим ребрам из этого множества. Стало быть, уже в $H_{1}$ не меньше $(x-1)(m-1)^{l}$ различных вершин степени не меньше $d$. Обозначим множество этих вершин через $U$.

Если $s=2 l$, то общее число ребер в $H_{1}$, а, значит, и в $H$, можно оценить снизу (используя формулы для $x$ и $d$ и оценку для $m$ ):

$$
\begin{gathered}
|E| \geqslant \frac{1}{n-l}(x-1)(m-1)^{l} d \geqslant \\
\geqslant \frac{n-2 l-1}{2(n-l)}\left(c_{0} r^{n-2 l}-1\right)^{l+1} \geqslant c^{l} r^{(n-2 l)(l+1)}=c^{\lfloor s / 2\rfloor} r^{(n-s)(\lfloor s / 2\rfloor+1)},
\end{gathered}
$$

где $c>0-$ некоторая подходящая малая константа. 
Если же $s=2 l+1$, то вершины из $U$ не могут иметь общих ребер, ведь все они находятся на расстоянии $l$ от $v$. В этом случае общее число ребер в $H_{1}$, а, значит, и в $H$, можно оценить снизу выражением

$$
|E| \geqslant(x-1)(m-1)^{l} d \geqslant c^{\lfloor s / 2\rfloor} n r^{(n-s)(\lfloor s / 2\rfloor+1)},
$$

где $c>0$ - это снова некоторая подходящая малая константа.

Вместе с (10) полученные оценки доказывают искомое утверждение следствия 2.

Дальнейшая структура работы следующая. В третьем разделе мы приводим формулировку Локальной леммы, которая необходима для доказательства теоремы 3. Четвертый раздел посвящен доказательству теоремы 3. В заключении мы обсуждаем усиления теоремы 3.

\section{3. Основные инструменты доказательства}

В качестве основного метода доказательства нами используется метод случайной перекраски. В нем мы следуем

- идеям Косточки и Кумбхата из [7] относительно двухэтапной перекраски,

- построению раскраски из работы Купавского и Шабанова [8],

- технике вероятностного анализа из работы Козика и Шабанова [4].

В последней из перечисленных работ использовался следующий вариант, так называемой, Локальной леммы, которая позволяет доказывать положительность вероятности одновременного невыполнения ряда событий в условиях слабой зависимости.

Теорема 4 (Локальная лемма). Пусть $X_{1}, \ldots, X_{N}$ - независимые случайные векторы, а $\mathcal{A}_{1}, \ldots, \mathcal{A}_{M}$ - собътия из алгебры, порожденной ими. Обозначим через $\operatorname{vln}\left(\mathcal{A}_{j}\right)$ такой минимальный набор векторов $X_{i}$, что $\mathcal{A}_{j} \in \sigma\left(X_{i}: i \in \operatorname{vln}\left(\mathcal{A}_{j}\right)\right)$. Введем для каљдого $i=1, \ldots, N$ следующие многочлены:

$$
w_{i}(z)=\sum_{\mathcal{A}: X_{i} \in v \ln (\mathcal{A})} \mathrm{P}(\mathcal{A}) z^{|v \ln (\mathcal{A})|} .
$$

Если существуют такой многочлен $w(z)$ и такое $\tau \in(0,1)$, что для любого $i=$ $1, \ldots, N$ и любого $z \geqslant 1$ выполнено $w_{i}(z) \leqslant w(z)$ и что

$$
w\left(\frac{1}{1-\tau}\right) \leqslant \tau,
$$

$\operatorname{mo} \mathrm{P}\left(\bigcap_{j=1}^{M} \overline{\mathcal{A}_{j}}\right)>0$.

Доказательство. Для каждого $j=1, \ldots, M$ положим $x_{j}=\mathrm{P}\left(\mathcal{A}_{j}\right)(1-\tau)^{-\left|v \ln \left(\mathcal{A}_{j}\right)\right|}$ и $\left.S_{j}=\left\{l: v \ln \left(\mathcal{A}_{l}\right) \cap \operatorname{vln}\left(\mathcal{A}_{j}\right) \neq \varnothing\right)\right\} \backslash\{j\}$. Тогда по определению $\operatorname{vln}\left(\mathcal{A}_{j}\right)$ событие $\mathcal{A}_{j}$ независимо с алгеброй событий $\left(\mathcal{A}_{l}, l \notin S_{j} \cup\{j\}\right)$. Для применения обычного варианта Локальной леммы (см., например, главу 5 в [9]) осталось показать, что

$$
\mathrm{P}\left(\mathcal{A}_{j}\right) \leqslant x_{j} \prod_{l \in S_{j}}\left(1-x_{l}\right) .
$$


Ho

$$
\begin{gathered}
x_{j} \prod_{l \in S_{j}}\left(1-x_{l}\right)=x_{j} \prod_{\mathcal{A}_{l}: v \ln \left(\mathcal{A}_{l}\right) \cap v \ln \left(\mathcal{A}_{j}\right) \neq \varnothing}\left(1-x_{l}\right) \geqslant x_{j} \prod_{X_{i} \in v \ln \left(\mathcal{A}_{j}\right)} \prod_{\mathcal{A}_{l}: X_{i} \in \mathcal{A}_{l}}\left(1-x_{l}\right) \geqslant \\
\geqslant x_{j} \prod_{X_{i} \in v \ln \left(\mathcal{A}_{j}\right)}\left(1-\sum_{\mathcal{A}_{l}: X_{i} \in \mathcal{A}_{l}} x_{l}\right)=x_{j} \prod_{X_{i} \in v \ln \left(\mathcal{A}_{j}\right)}\left(1-w_{i}\left(\frac{1}{1-\tau}\right)\right) \geqslant \\
\geqslant x_{j} \prod_{X_{i} \in v \ln \left(\mathcal{A}_{j}\right)}\left(1-w\left(\frac{1}{1-\tau}\right)\right)=x_{j}\left(1-w\left(\frac{1}{1-\tau}\right)\right)^{\left|v \ln \left(\mathcal{A}_{j}\right)\right|} \geqslant \\
\geqslant x_{j}(1-\tau)^{\left|v \ln \left(\mathcal{A}_{j}\right)\right|}=\mathrm{P}\left(\mathcal{A}_{j}\right) .
\end{gathered}
$$

В настоящей статье мы будем всегда работать с $n$-однородными гиперграфами и полагать $\tau=\frac{1}{n+1}$.

\section{4. Доказательство теоремы 3}

Итак, пусть $n$-однородный гиперграф $H=(V, E)$ удовлетворяет условиям теоремы 3. Нам необходимо доказать существование правильной раскраски множества вершин гиперграфа $H$ в $r$ цветов. Для этого мы построим некоторую случайную $r$-раскраску $V$ и покажем, что она является правильной с положительной вероятностью. Без ограничения общности будем считать, что $V=\{1, \ldots, N\}$. Также всюду далее $\delta$-тяжелые ( $\delta$-легкие) вершины и ребра будем называть просто тяжельми и легкими. Наконец, для каждого ребра $A$ через $A_{R}$ мы будем обозначать множество его тяжелых вершин, а через $A_{L}-$ множество его легких вершин.

4.1. Построение случайной раскраски Рассмотрим следующий набор случайных величин.

(1) $\left\{\xi_{1}, \ldots, \xi_{N}\right\}$ - независимые случайные величины с равномерным распределением на множестве цветов $\{1, \ldots, r\}$.

(2) $\left\{\eta_{1}, \ldots, \eta_{N}\right\}$ - независимые случайные величины со следующим условным распределением на множестве цветов $\{1, \ldots, r\}$ :

$$
\mathrm{P}\left(\eta_{v}=\alpha \mid \xi_{v}=\beta\right)=\frac{1}{r-1} \text { для любых } \alpha \neq \beta \in\{1, \ldots, r\} \text {, }
$$

т.е. $\eta_{v}$ имеет равномерное условное распределение на множестве $\{1, \ldots, r\} \backslash$ $\left\{\xi_{v}\right\}$.

(3) $X_{1}, \ldots, X_{N}$ - независимые (независимые также с набором $\left(\xi_{v}, \eta_{v}, v \in V\right)$ ) случайные величины с равномерным распределением на отрезке $[0,1]$.

Наше построение случайной раскраски и ее анализ основаны на методе случайной перекраски. Опишем конструкцию с помощью следующего рандомизированного алгоритма.

- Стартуем с равномерной случайной раскраски множества вершин в $r$ цветов: вершине $v$ присваиваем цвет $\xi_{v}$. 
- В случайной раскраске $\xi=\left(\xi_{1}, \ldots, \xi_{N}\right)$ гиперграф $H$ может содержать одноиветные и почти одноцветные ребра. Ребро $A$ называется почти одночветным с доминирующим иветом $\alpha$ в раскраске $\xi$, если в нем почти все вершины покрашены в цвет $\alpha$, а именно, если

$$
n-s \leqslant \sum_{v \in A} I\left\{\xi_{v}=\alpha\right\} \leqslant n-1,
$$

где $1 \leqslant s<n / 2-$ первый параметр нашей конструкции.

- Будем осуществлять следующий алгоритм случайной перекраски, в рамках которого мы хотели бы исправить одноцветные ребра, но так, чтобы почти одноцветные ребра не стали одноцветными доминирующего цвета. Зададим случайный порядок вершин $\sigma$ с помощью вариационного ряда случайных величин $\left\{X_{v}, v \in V\right\}$. Для ребра $A$ и вершины $v$ будем использовать обозначение $\sigma(v, A)$ для номера вершины $v$ в ребре $A$. Формально:

$$
\sigma(v, A)=\sum_{w \in A} I\left\{X_{w} \leqslant X_{v}\right\}
$$

- На первом этапе процесса случайной перекраски будем перекрашивать только тяжелые вершины гиперграфа $H$ и исправлять только тяжелые одноцветные ребра. Будем рассматривать тяжелые вершины в порядке, заданном $\sigma$, и для текущей вершины $v$ проверять следующие условия перекраски:

1h во-первых, существует такое тяжелое ребро $A, v \in A$, что $A$ одноцветно в $\xi$, на момент рассмотрения $v$ ни одна вершина $A$ еще не сменила цвет и, кроме того, $\sigma\left(A_{R}, v\right) \leqslant h$ (т.е. вершина $v$ должна иметь номер не более $h$ среди всех тяжелых вершин ребра $A$, здесь $1 \leqslant h<n / 2-$ второй параметр конструкции);

$\mathbf{2 h}$ во-вторых, не существует такого тяжелого ребра $B, v \in B$, почти одноцветного в $\xi$, что на момент рассмотрения $v$ эта вершина осталась единственной, не покрашенной в доминирующий цвет $\alpha$ ребра $B$, и $\eta_{v}=\alpha$.

- Если условие перекраски выполнено, то присваиваем вершине $v$ цвет $\eta_{v} . \mathrm{B}$ противном случае, не перекрашиваем $v$ и переходим к следующей вершине.

Получившуюся в результате перекраски тяжелых вершин случайную раскраску обозначим через $\zeta=\left(\zeta_{1}, \ldots, \zeta_{N}\right)$. Во время второго этапа перекраски мы будем перекрашивать только легкие вершины.

- Будем рассматривать легкие вершины в порядке, заданном $\sigma$, и для текущей вершины $w$ проверять следующие условия перекраски:

11 во-первых, существует такое легкое ребро $A, w \in A$, что $A$ одноцветно или почти одноцветно в $\xi$, одноцветно в $\zeta$, на момент рассмотрения $w$ ни одна легкая вершина $A$ еще не сменила цвет и, кроме того, $\sigma\left(A_{L}, v\right) \leqslant h$;

$2 \mathbf{l}$ во-вторых, не существует такого ребра $B, w \in B$, почти одноцветного в $\xi$, что на момент рассмотрения $w$ эта вершина осталась единственной, не покрашенной в доминирующий цвет $\alpha$ ребра $B$, и $\eta_{w}=\alpha$.

- Если условие перекраски выполнено, то присваиваем вершине $v$ цвет $\eta_{v}$. В противном случае, не перекрашиваем $v$ и переходим к следующей вершине.

Наша задача состоит в том, чтобы показать, что в условиях теоремы при подходящем выборе параметров $s, h$ с положительной вероятностью: 
(1) после первого этапа перекраски все одноцветные в $\xi$ тяжелые ребра перестанут быть одноцветными того же цвета ,

(2) после второго этапа перекраски все одноцветные в $\zeta$ легкие ребра, которые были одноцветными того же цвета или почти одноцветными с тем же доминирующим цветом в начальной раскраске $\xi$, будут исправлены,

(3) в итоговой раскраске не появится одноцветных ребер, которые в $\xi$ не были одноцветными того же цвета или почти одноцветными с тем же доминирующим цветом.

В следующем параграфе мы осуществим построение так называемого дерева зависимостей между ребрами гиперграфа в процессе перекраски.

4.2. Построение дерева зависимостей Проанализируем возможности для смены цвета каждой вершиной в процессе перекраски.

Первый этап, тяжелые вершины и ребра. Согласно условию $1 \mathbf{h}$ нашего алгоритма, тяжелая вершина $v$ может сменить свой цвет только на первом этапе. При этом должно существовать такое тяжелое ребро $A$, содержащее $v$, которое являлось одноцветным (например, цвета $\alpha$ ) в начальной раскраске $\xi$, на момент рассмотрения $v$ оно все еще одноцветно цвета $\alpha$ и $v$ имеет номер не более $h$ в этом ребре среди всех тяжелых вершин. В этом случае будем говорить, что ребро $A$ влияет на вершину $v$. Ясно, что каждое ребро может влиять только на одну вершину - первую вершину, сменившую цвет в ребре.

Однако второе условие $\mathbf{2 h}$ в некоторых случаях запрещает перекраску вершины $v$ при выполнении первого условия $\mathbf{1 h}$. В этом случае должно существовать такое тяжелое ребро $B$, также содержащее $v$, которое являлось почти одноцветным (например, с доминирующим цветом $\beta$ ) в начальной раскраске $\xi$ и на момент рассмотрения $v$ только эта вершина осталась не покрашенной в цвет $\beta$. В такой ситуации будем говорить, что ребро $B$ блокирует перекраску вершины $v$. Заметим, что каждое ребро может блокировать ровно одну вершину, а также в указанной ситуации должно быть выполнено $\eta_{v}=\beta$ (иначе смена цвета не превращала бы $B$ в одноцветное ребро).

Пусть теперь тяжелое ребро $A$ было одноцветным цвета $\alpha$ и после первого этапа перекраски таким же и осталось, т.е. ни одна вершина $A$ не сменила свой цвет. Тогда мы можем построить дерево из ребер гиперграфа $H$ с корнем $A$ следующим образом.

а) Пусть $v_{1}, \ldots, v_{h}$ - первые $h$ тяжелых вершин этого ребра. В силу того, что первое условие на перекраску для каждой из них было выполнено, но цвет они так и сменили, их перекраска была заблокирована почти одноцветными тяжелыми ребрами $B_{1}, \ldots, B_{h}$ с доминирующими цветами $\beta_{1}, \ldots, \beta_{h}$. Эти ребра назовем потомками ребра $A$ в нашем дереве зависимостей.

б) Если в начальной раскраске $\xi$ только вершина $v_{i}$ не была покрашена в цвет $\beta_{i}$, то ветвь ребра $B_{i}$ закончена. Если же нет, то пусть $v_{i, 1}, \ldots, v_{i, l_{i}}, l_{i} \leqslant s-1$, другие вершины ребра $B_{i}$, покрашенные изначально не в доминирующий цвет $\beta_{i}$. Тогда к моменту рассмотрения $v_{i}$ все эти вершины должны были сменить цвет на $\beta_{i}$. Значит, для каждой вершины $v_{i, j}$ найдется тяжелое ребро $C_{i, j}$, одноцветное не цвета $\beta_{i}$, которое повлияло на вершину $v_{i, j}$. Ребра $C_{i, j}, j \leqslant l_{i}$, назовем потомками ребра $B_{i}$ в дереве зависимостей. 
в) Если $\sigma\left(v_{i, j},\left(C_{i, j}\right)_{R}\right)=1$, то ветвь ребра $C_{i, j}$ закончена. В противном случае пусть $v_{i, j}^{t}, t=1, \ldots, \sigma\left(v_{i, j}, C_{i, j}\right)-1$, - те вершины $C_{i, j}$, имеющие меньший номер, чем $v_{i, j}, X_{v_{i, j}^{1}}<X_{v_{i, j}^{2}}<\ldots<X_{v_{i, j}}$. Для них проводим те же рассуждения, что и в п. а) для вершин $v_{1}, \ldots, v_{h}$.

Повторяем процедуру, пока не закончится набор каждой ветви дерева с корнем $A$ из ребер гиперграфа $H$. В результате мы получим дерево зависимостей $\mathcal{T}$ с корнем $A$ и со следующими свойствами.

(1) Вершинами этого дерева являются тяжелые ребра $H$, которые являются одноцветными или почти одноцветными в начальной раскраске $\xi$.

(2) Если две вершины $C, B$ образуют ребро в $\mathcal{T}$, то, как ребра гиперграфа $H$ они должны иметь общий элемент $v=C \cap B$, который мы будем называть узловой вершиной дерева $\mathcal{T}$. При этом одно из этих ребер (например, $C$ ) должно быть одноцветным цвета $\alpha$ в $\xi$, а второе $(B)$ - почти одноцветным цвета $\beta \neq \alpha$. Если $C$ находится ближе к корню дерева, то тогда $B$ блокирует перекраску вершины $v$. В этом случае назовем $v$ узловой вершиной первого типа. Если же, наоборот, $B$ находится ближе к корню, то тогда $C$ должно влиять на $v$, и $v$ называется узловой вершиной второго типа. Кроме того, в обоих случаях должно быть выполнено $\eta_{v}=\beta$.

(3) Одноцветные ребра имеют четное реберное расстояние до корня $\mathcal{T}$, почти одноцветные - нечетное. Для вершины $A$ дерева $\mathcal{T}$ обозначим через $\rho(A)$ его расстояние до корня.

(4) Степень каждой вершины $\mathcal{T}$, являющейся почти одноцветным ребром, совпадает с количеством его вершин, покрашенных в $\xi$ не в доминирующий цвет, и, значит, не превосходит $s$. При этом каждая такая вершина является узловой.

(5) Степень каждой вершины $\mathcal{T}$, являющейся одноцветным ребром в $\xi$, не превосходит $h$. При этом узловые вершины этого ребра - это в точности его первые по нумерации $\sigma$ тяжелые вершины.

(6) Степень корня всегда равна $h$.

Будем называть $\mathcal{T}^{\prime}$ деревом, если

(1) $\mathcal{T}^{\prime}=\left(V^{\prime}, E^{\prime}, v\right)$, где $V^{\prime}$ - множество вершин $\mathcal{T}^{\prime}, E^{\prime}-$ множество ребер $\mathcal{T}^{\prime}$, $v \in V^{\prime}-$ корень $\mathcal{T}^{\prime}$

(2) пара $\left(V^{\prime}, E^{\prime}\right)$ является ацикличным связным графом;

(3) множество вершин дерева $V^{\prime}$ является подмножеством множества ребер гиперграфа $H$.

Обозначим через $\mathcal{A}\left(\mathcal{T}^{\prime}\right)$ событие, состоящее в том, что дерево $\mathcal{T}^{\prime}$ является деревом зависимостей на первом этапе перекраски.

Второй этап, легкие вершины. На втором этапе перекраски мы осуществляем перекраску легких вершин. Здесь многое аналогично. Легкая вершина $v$ может сменить свой цвет согласно 1l, если существует такое легкое ребро $A$, содержащее $v$, что $A$ было одноцветным (некоторого цвета $\alpha$ ) в промежуточной раскраске $\zeta$, а в начальной раскраске $\xi$ оно было либо одноцветным цвета $\alpha$, либо почти одноцветным с доминирующим цветом $\alpha$. Тогда ребро $A$ влияет на вершину $v$.

Второе же условие $2 \mathbf{l}$ в некоторых случаях запрещает перекраску легкой вершины $v$. В этом случае должно существовать такое ребро $B$ (неважно, тяжелое или легкое), также содержащее $v$, которое являлось почти одноцветным (например, с доминирующим цветом $\beta$ ) в начальной раскраске $\xi$ и на момент рассмотрения $v$ только 
эта вершина осталась не покрашенной в цвет $\beta$. В такой ситуации будем говорить, что ребро $B$ блокирует перекраску легкой вершины $v$. Заметим, что также должно быть выполнено $\eta_{v}=\beta$, иначе смена цвета не превращала бы $B$ в одноцветное ребро.

Пусть теперь легкое ребро $A$ является одноцветным цвета $\alpha$ в итоговой раскраске (после всех этапов перекраски), и при этом после первого этапа оно также было одноцветным цвета $\alpha$. Тогда в начальной раскраске оно было либо одноцветным цвета $\alpha$, либо почти одноцветным с доминирующим цветом $\alpha$. Построение дерева зависимостей проходит аналогично построению на первом этапе перекраски, но есть некоторые отличия.

а) Пусть $v_{1}, \ldots, v_{h}$ - первые $h$ легких вершин этого ребра. В силу того, что первое условие на перекраску для каждой из них было выполнено, но цвет они так и не сменили, их перекраска была заблокирована почти одноцветными ребрами $B_{1}, \ldots, B_{h}$ с доминирующими цветами $\beta_{1}, \ldots, \beta_{h}$. Эти ребра мы назовем новыми потомками ребра $A$. Заметим, что в начальной раскраске $\xi$ ребро $A$ могло быть неодноцветным, а лишь почти одноцветным цвета $\alpha$. В этом случае вершины, которые не были покрашены в доминирующий цвет, должны были сменить свой цвет на $\alpha$ на первом этапе процесса перекраски. Это означает, что все эти вершины $w_{1}, \ldots, w_{k}, k \leqslant s$, являются тяжелыми и что есть набор тяжелых ребер $C_{1}, \ldots, C_{k}$, повлиявших на них. Эти ребра мы назовем старыми потомками ребра $A$.

б) Дальнейший анализ ветвей, начинающихся с ребер $C_{1}, \ldots, C_{k}$, является полностью таким же, как и в предыдущем случае, ведь здесь перекрашиваются только тяжелые вершины. Все потомки будут соединяться через тяжелые вершины, а потому мы их будем называть старьми.

в) Далее, если в начальной раскраске $\xi$ только вершина $v_{i}$ не была покрашена в цвет $\beta_{i}$, то и ветвь ребра $B_{i}$ закончена. Если же нет, то пусть $v_{i, 1}, \ldots, v_{i, l_{i}}, l_{i} \leqslant$ $s-1$, - другие вершины ребра $B_{i}$, покрашенные изначально не в доминирующий цвет $\beta_{i}$. Тогда к моменту рассмотрения $v_{i}$ все эти вершины должны были сменить цвет на $\beta_{i}$. Значит, для каждой вершины $v_{i, j}$ найдется либо тяжелое (если $v_{i, j}-$ тяжелая вершина), либо легкое (если $v_{i, j}$ - легкая вершина) ребро $C_{i, j}$, одноцветное не цвета $\beta_{i}$, которое повлияло на вершину $v_{i, j}$. В первом случае $C_{i, j}$ назовем старым потомком $B_{i}$, а во втором - новъм.

г) Если $v_{i, j}$ - тяжелая вершина, то анализ ветви $C_{i, j}$ будет тем же, что и на первом этапе. Здесь будут только старые потомки. В случае же легкого ребра $C_{i, j}$ возможны как новые, так и старые потомки. Анализ таков же, как и анализ ребра $A$.

Повторяем процедуру, пока не закончится набор каждой ветви дерева с корнем $A$ из ребер гиперграфа $H$. В результате мы получим дерево зависимостей $\mathcal{T}$ с корнем $A$ и со следующими свойствами.

(1) Вершинами этого дерева являются легкие и тяжелые ребра $H$, которые являются одноцветными или почти одноцветными в начальной раскраске $\xi$.

(2) Если две вершины $C, B$ образуют ребро в $\mathcal{T}$, то, как ребра гиперграфа $H$ они должны иметь общий элемент $v=C \cap B$, который мы будем называть узловой вершиной дерева $\mathcal{T}$. Классификация вершин в этом случае является более сложной. Возможны следующие варианты. Будем считать, что $C$ находится ближе к корню, чем $B$.

- Вершина $v$ является тяжелой, ребро $B$ - тяжелым одноцветным в $\xi$, а $C$ - почти одноцветным легким в $\xi$. Это узловая вершина нулевого тuna. 
- Вершина $v$ является тяжелой, ребро $B$ - тяжелым одноцветным в $\xi$, а $C$ - почти одноцветным тяжелым в $\xi$. Это узловая вершина первого muna.

- Вершина $v$ является тяжелой, ребро $B$ - тяжелым почти одноцветным в $\xi$, а $C$ - тяжелым одноцветным в $\xi$. Это узловая вершина второго muna.

- Вершина $v$ является легкой, ребро $B$ - одноцветным легким в $\zeta$, а ребро $C$ - почти одноцветным (легким или тяжелым) в $\xi$. Это узловая вершина mретвего muna.

- Вершина $v$ является легкой, ребро $C$ - одноцветным легким в $\zeta$, а ребро $B$ - почти одноцветным (легким или тяжелым) в $\xi$. Это узловая вершина четвертого типа.

В первом и третьем случаях ребро $B$ влияет на вершину $v$, а во втором и четвертом - блокирует его перекраску. В одном случае значение $\eta_{v}$ должно быть равно доминирующему цвету $C$, а в другом - доминирующему цвету $B$.

(3) Степень каждой вершины $\mathcal{T}$ не превосходит $h+s$.

(4) Степень корня всегда не меньше $h$.

Обозначим через $\mathcal{C}\left(\mathcal{T}^{\prime}\right)$ событие, состоящее в том, что дерево $\mathcal{T}^{\prime}$ является деревом зависимостей на втором этапе перекраски.

Дальнейший вероятностный анализ зависит от того, образует ли набор вершин $\mathcal{T}^{\prime}$, как набор ребер гиперграфа $H$, гипердерево или нет.

4.3. Анализ первого этапа процесса перекраски Пусть $\mathcal{T}$ - дерево зависимостей размера $T$ (то есть число вершин в дереве $\mathcal{T}$ равно $T$ ) на первом этапе перекраски. Оценим вероятность события $\mathcal{A}(\mathcal{T})$ в зависимости от реальной структуры дерева.

Первый случай: гипердерево. Пусть набор вершин дерева $\mathcal{T}$ образует гипердерево размера $T$ в $H$. Тогда одноцветные ребра нашего дерева не пересекаются, а с почти одноцветными ребрами они пересекаются только по узловым вершинам и только при условии соседства в $\mathcal{T}$.

Пусть $A_{1}, \ldots, A_{T}$ - ребра гиперграфа $H$, они же являются вершинами $\mathcal{T}$. Если $\mathcal{T}$ является деревом зависимостей, то каждое $A_{i}$ является либо одноцветным цвета $\alpha_{i}$ ребром $H$ в раскраске $\xi$, либо почти одноцветным ребром с доминирующим цветом $\alpha_{i}$ в раскраске $\xi$. Тогда при фиксированных $\alpha_{1}, \ldots, \alpha_{T}$ однозначно определён цвет любой вершины гипердерева в начальной раскраске $\xi$, ведь либо вершина лежит в одноцветном ребре, либо является неузловой вершиной почти одноцветного ребра и, стало быть, должна быть покрашена в его доминирующий цвет. Далее, для каждой узловой вершины $v$ будет однозначно определено значение $\eta_{v}$. Наконец, номер узловой вершины, принадлежащей одноцветному ребру $A$, среди тяжёлых вершин этого ребра не может превосходить степени $A$ как вершины дерева $\mathcal{T}$.

Обозначим через $\mathcal{A}_{1}(\mathcal{T})$ событие $\mathcal{A}(\mathcal{T})$ в случае, если рассматриваемая конфигурация ребер гиперграфа является гипердеревом. Тогда вероятность подобного события оценивается следующим образом:

$$
\begin{gathered}
\mathrm{P}\left(\mathcal{A}_{1}(\mathcal{T}) \mid \alpha_{1}, \ldots, \alpha_{T}\right)= \\
=\left(\frac{1}{r}\right)^{T(n-1)+1} \frac{1}{(r-1)^{T-1}} \prod_{i: \rho\left(A_{i}\right)=0(\bmod 2)}\left[\frac{1}{\left(\left|\left(A_{i}\right)_{R}\right|\right)_{\operatorname{deg}\left(A_{i}\right)}}\right],
\end{gathered}
$$


где через $\operatorname{deg}\left(A_{i}\right)$ обозначена степень вершины $A_{i}$ в дереве $\mathcal{T}$, а через $(a)_{b}$ обозначено число размещений без повторений, $(a)_{b}=a(a-1) \ldots(a-b+1)$.

Упростим немного формулу (11). Заметим, во-первых, что $\operatorname{deg}\left(A_{i}\right) \leqslant h$ для одноцветного ребра $A_{i}$. Далее, всего в конфигурации из $T$ ребер есть ровно $T-1$ узловых вершин, каждая из которых содержится ровно в одном одноцветном и ровно в одном почти одноцветном ребре. Отсюда

$$
\sum_{i: \rho\left(A_{i}\right)=0(\bmod 2)} \operatorname{deg}\left(A_{i}\right)=T-1 .
$$

Значит, выражение в (11) не превосходит

$$
\begin{gathered}
\left(\frac{1}{r}\right)^{T(n-1)+1} \frac{1}{(r-1)^{T-1}} \prod_{i: \rho\left(A_{i}\right)=0(\bmod 2)}\left[\frac{1}{\left(n / 2-\operatorname{deg}\left(A_{i}\right)+1\right)^{\operatorname{deg}\left(A_{i}\right)}}\right] \leqslant \\
\leqslant\left(\frac{1}{r}\right)^{n T-T+1}\left(\frac{1}{(r-1)(n / 2-h)}\right)^{T-1} .
\end{gathered}
$$

Добавив выбор цветов $r(r-1)^{T-1}$ (цвета соседей не должны совпадать), получаем следующую оценку:

$\mathrm{P}\left(\mathcal{A}_{1}(\mathcal{T})\right) \leqslant r(r-1)^{T-1}\left(\frac{1}{r}\right)^{n T-T+1}\left(\frac{1}{(r-1)(n / 2-h)}\right)^{T-1}=\left(\frac{1}{r}\right)^{(n-1) T} \frac{1}{(n / 2-h)^{T-1}}$.

Теперь оценим вклад событий типа $\mathcal{A}_{1}$ в локальный многочлен. Локальную лемму (теорема 4) мы будем применять к случайным векторам $Y_{1}, \ldots, Y_{N}$, где $Y_{j}=\left(\xi_{j}, \eta_{j}, X_{j}\right)$. Легко понять, что для дерева зависимостей размера $T$ выполнено

$$
v \ln \left(\mathcal{A}_{1}(\mathcal{T})\right)=n T-n+1 \leqslant n T
$$

т.е. просто равно числу вершин гиперграфа в конфигурации.

Оценим число деревьев зависимостей, в которых может поучаствовать фиксированная вершина $v$. Для этого мы сопоставим дереву зависимостей $\mathcal{T}$ обычное непомеченное дерево $t$ с корнем. Для каждого такого дерева зафиксируем нумерацию вершин $\tau(t)$, начиная с корня и продолжая по возрастанию расстояния от него (корень имеет номер 1 , его потомки $-2,3, \ldots$ и т.д.). Пусть $\mathcal{T}-$ гипердерево из $T$ тяжелых ребер, для которого выполнено событие $\mathcal{A}_{1}(\mathcal{T})$. В рамках этого события все потомки одноцветных ребер имеют свой внутренний номер, отвечающий номеру вершины в его пересечении с родителем. Например, пусть ребра $A_{1}, \ldots, A_{x}$ - потомки одноцветного ребра $A, v_{i}=A \cap A_{i}$. Тогда если $\sigma\left(A, v_{1}\right)<\ldots<\sigma\left(A, v_{x}\right)$, то ребро $A_{1}$ имеет внутренний номер $1, A_{2}-$ номер 2 и т.д.

Тогда сопоставление дерева $t$ гипердереву $\mathcal{T}$ должно соблюдать внутреннюю нумерацию у потомков каждого одноцветного ребра. Так если внутренний номер $A_{1}$ меньше номера $A_{2}$, то соответствующие им узлы $u_{1}, u_{2}$ дерева $t$ должны идти в том же порядке по нумерации $\tau(t)$.

Дерево $t$ размера $T$ можно выбрать не более чем $4^{T} / T$ способами. Тяжелое ребро $+A_{1}$, содержащее $v$, можно выбрать не более чем $\Delta=\Delta(H)$ способами. Место ребра $A_{1}$ в дереве зависимостей можно выбрать не более чем $T$ способами. Далее, каждое следующее ребро в конфигурации гиперграфа можно выбрать не более чем 
$n \delta$ способами (мы заполняем дерево так: сначала доходим до корня, а затем по возрастанию номеров вершин в дереве), ведь мы должны сначала выбрать тяжелую вершину в предыдущем ребре-соседе, а затем - тяжелое ребро ее содержащее. Наконец, размер $T$ должен быть не меньше $h+1$. Учитывая (8), (9), получаем следующую оценку вклада событий этого типа в локальный многочлен:

$$
\begin{gathered}
w_{1}(z) \leqslant \sum_{T=h+1}^{+\infty} \frac{4^{T}}{T} \Delta T(n \delta)^{T-1}\left(\frac{1}{r}\right)^{(n-1) T} \frac{1}{(n / 2-h)^{T-1}} z^{n T} \leqslant \\
\leqslant \sum_{T=h+1}^{+\infty}\left(4 z^{n}\right)^{T} n^{\alpha} c^{T-1}\left(\frac{n}{n / 2-h}\right)^{T-1} .
\end{gathered}
$$

При $z=z_{0}=1 /(1-\tau), \tau=1 /(n+1)$, и $h<n / 4$, имеем

$$
w_{1}\left(z_{0}\right) \leqslant \sum_{T=h+1}^{+\infty}(4 e)^{T} n^{\alpha} c^{T-1} 4^{T-1} \leqslant 4 e n^{\alpha} \sum_{T=h+1}^{+\infty}(16 e c)^{T-1} \leqslant
$$

(при $16 e c<1)$

$$
\leqslant \frac{4 e n^{\alpha}}{1-16 e c}(16 e c)^{h}
$$

Второй случай: гиперциклы. Рассмотрим теперь случай, когда из ребер гиперграфа, входящих в дерево зависимостей $\mathcal{T}$, можно составить гиперцикл. Выберем минимальное по размеру поддерево $\mathcal{T}^{\prime}$, содержащее гиперцикл. Под поддеревом мы понимаем вершину дерева (корень поддерева) и всех ее потомков. Все прямые поддеревья $\mathcal{T}^{\prime}$, т.е. поддеревья, корнями которых являются потомки корня $\mathcal{T}^{\prime}$, являются гипердеревьями в силу минимальности $\mathcal{T}^{\prime}$. Введем также некоторый параметр $q$. Тогда возможны следующие варианты.

- Нашлось прямое поддерево зависимостей размера не менее $q$.

- Все прямые поддеревья имеют размер не более $q-1$. Тогда найдутся два поддерева, которые пересекаются по вершинам. Вместе с корнем $\mathcal{T}^{\prime}$ получится цикл длины не более $2 q$. Значит, найдется и простой цикл длины не более $2 q$.

Проанализируем первый вариант. В нем мы снова имеем дерево зависимостей $\mathcal{T}$ размера не менее $q$, единственное отличие - это то, что при фиксированных цветах ребер $\alpha_{1}, \ldots, \alpha_{|\mathcal{T}|}$ (для одноцветного ребра - это его цвет в $\xi$, для почти одноцветного - его доминирующий цвет в $\xi$ ) мы знаем все изначальные цвета вершин в корне дерева $A_{1}$ за исключением одной вершины, через которую она "присоединяется" к своему предку в полном дереве зависимостей. Для подобной вершины $w$ должно быть выполнено $\xi_{w} \neq \alpha_{1}$, а $\eta_{w}=\alpha_{1}$. Стало быть, вероятность подобного события $\mathcal{A}_{2}(\mathcal{T})$ можно оценить, как и вероятность $\mathcal{A}_{1}(\mathcal{T})($ см. $(11))$, только умноженную на $n$ (число вариантов выбора особой вершины $w$ в корне). Вклад же таких событий в локальный многочлен тоже можно оценить аналогичным образом при $z=z_{0}=1+1 / n$, учитывая (13),

$$
w_{2}\left(z_{0}\right) \leqslant \sum_{T=q}^{+\infty} \frac{4^{T}}{T} \Delta T(n \delta)^{T-1}\left(\frac{1}{r}\right)^{(n-1) T} \frac{1}{(n / 2-h)^{T-1}} n z_{0}^{n T} \leqslant \frac{4 e n^{\alpha+1}}{1-16 e c}(16 e c)^{q-1} .
$$


Осталось разобраться с простым циклом. Пусть $\left(A_{1}, \ldots, A_{t}\right)$ - полученный простой цикл. Заметим, что каждое из ребер является либо почти одноцветным, либо одноцветным в начальной раскраске $\xi$. Пусть $\alpha_{1}, \ldots, \alpha_{t}$ - доминирующие цвета этих ребер в $\xi$. Тогда в каждом ребре $A_{j}$ найдется не более $s$ вершин окрашенных цветом $\alpha_{j}$ в $\xi$, однако $\eta_{w}=\alpha_{j}$ для каждой такой вершины $w \in A_{j}$, что $\xi_{w} \neq \alpha_{j}$. Поэтому должно быть выполнено событие

$$
\mathcal{B}\left(A_{j}\right)=\left(\bigcap_{w \in A_{j}}\left\{\left\{\xi_{w}=\alpha_{j}\right\} \cup\left\{\xi_{w} \neq \alpha_{j}, \eta_{w}=\alpha_{j}\right\}\right\}\right) \cap\left\{\sum_{w \in A_{j}} I\left\{\xi_{w} \neq \alpha_{j}\right\} \leqslant s\right\} .
$$

В силу того, что цикл простой, каждое ребро содержит $n-2$ вершины, не содержащиеся в других ребрах цикла. Стало быть, обозначив через $\mathcal{B}\left(A_{1}, \ldots, A_{t}\right)$ пересечение событий $\mathcal{B}\left(A_{j}\right)$, получаем:

$$
\begin{gathered}
\mathrm{P}\left(\mathcal{B}\left(A_{1}, \ldots, A_{t}\right) \mid \alpha_{1}, \ldots, \alpha_{t}\right) \leqslant\left(\sum_{i=0}^{s}\left(\begin{array}{c}
n-2 \\
i
\end{array}\right)\left(\frac{1}{r}\right)^{n-2}\right)^{t} \leqslant \\
\leqslant r^{-(n-2) t} n^{s t}\left(\sum_{i=0}^{s} \frac{1}{i !}\right)^{t} \leqslant r^{-(n-2) t} n^{s t} e^{t} .
\end{gathered}
$$

Учитывая выбор цветов, имеем

$$
\mathrm{P}\left(\mathcal{B}\left(A_{1}, \ldots, A_{t}\right)\right) \leqslant r^{3 t-n t} n^{s t} e^{t} .
$$

Осталось оценить вклад данного типа событий в локальный полином. Для каждой вершины $v$ содержащее ее ребро цикла можно выбрать не более чем $\Delta$ способами. Далее, номер ребра $j$ в цикле можно выбрать не более чем $t$ способами. Если номер строго меньше $t$, то ребра $A_{1}, \ldots, A_{t-1}$, кроме $j$, можно выбрать не более чем $n \Delta$ способами. Ребро же $A_{t}$ должно пересекать, как $A_{1}$, так и $A_{t-1}$, поэтому в силу простоты гиперграфа $H$ такое ребро можно выбрать не более чем $n^{2}$ способами, ведь каждая пара вершин, одна из $A_{1}$, вторая из $A_{t-1}$, однозначно определяет подобное ребро. Если же $j=t$, то мы сначала выбираем $A_{2}, \ldots, A_{t-1}$ не более чем $(n \Delta)^{t-2}$ способами, а ребро $A_{1}$ - не более чем $n^{2}$ способами.

В итоге получаем следующую оценку вклада событий $\mathcal{B}\left(A_{1}, \ldots, A_{t}\right)$ в локальный полином при $z=z_{0}=1+1 / n$, используя (8) во втором переходе:

$$
\begin{gathered}
w_{3}\left(z_{0}\right) \leqslant \sum_{t=3}^{2 q} t \Delta(n \Delta)^{t-2} r^{3 t-n t} n^{s t} e^{t} z_{0}^{n t} \leqslant \\
\leqslant \sum_{t=3}^{2 q} t n^{(\alpha+s) t} r^{2 t-n+1} e^{2 t} \leqslant 2 q e^{4 q} n^{2 q(\alpha+s)} r^{2 q+1-n} .
\end{gathered}
$$

На этом анализ первого этапа перекраски закончен.

4.4. Анализ второго этапа перекраски Пусть $\mathcal{T}$ - дерево зависимостей размера $T$ на втором этапе перекраски. Оценим вероятность события $\mathcal{C}(\mathcal{T})$ в зависимости от реальной структуры дерева. Как и в анализе первого этапа, рассмотрим сначала случай, когда ребра гиперграфа, входящие в $\mathcal{T}$, образуют гипердерево.

Оценка вероятности подобного события $\mathcal{C}(\mathcal{T})$ будет почти такой же, как и на первом этапе. Действительно, пусть снова $A_{1}, \ldots, A_{T}$ - ребра гиперграфа, вершины $\mathcal{T}$. 
Пусть $\alpha_{1}, \ldots, \alpha_{T}-$ соответствующий им набор цветов (для одноцветного ребра это его цвет в $\xi$, для почти одноцветного - его доминирующий цвет в $\xi$ ). Тогда при фиксированных $\alpha_{1}, \ldots, \alpha_{T}$ однозначно определен цвет любой вершины гипердерева в начальной раскраске $\xi$, ведь либо вершина лежит в одноцветном ребре, либо является неузловой вершиной почти одноцветного ребра и, стало быть, должна быть покрашена в его доминирующий цвет. Далее, для каждой узловой вершины $v$ определено значение $\eta_{v}$. Наконец, каждая узловая вершина имеет номер не более $\operatorname{deg}(A)$ среди вершин того же типа в содержащем ее одноцветном ребре $A$ (для тяжелых вершин - одноцветное в $\xi$ тяжелое ребро, а для легких - одноцветное в $\zeta$ легкое ребро). Тогда, учитывая, что степень каждой вершины дерева не превосходит $h+s$, вероятность рассматриваемого события $\mathcal{C}(\mathcal{T})$ оценивается следующим образом:

$$
\mathrm{P}\left(\mathcal{C}(\mathcal{T}) \mid \alpha_{1}, \ldots, \alpha_{T}\right) \leqslant\left(\frac{1}{r}\right)^{T(n-1)+1} \frac{1}{(r-1)^{T-1}}\left(\frac{1}{n / 2-h-s}\right)^{T-1} .
$$

Добавив выбор цветов $r(r-1)^{T-1}$ способами (цвета соседей не должны совпадать), получаем, что

$$
\begin{gathered}
\mathrm{P}(\mathcal{C}(\mathcal{T})) \leqslant r(r-1)^{T-1}\left(\frac{1}{r}\right)^{n T-T+1}\left(\frac{1}{(r-1)(n / 2-h-s)}\right)^{T-1}= \\
=\left(\frac{1}{r}\right)^{(n-1) T} \frac{1}{(n / 2-h-s)^{T-1}} .
\end{gathered}
$$

Теперь оценим вклад в локальный полином. Дерево $t$ размера $T$ можно выбрать не более чем $4^{T} / T$ способами. Каждое ребро дерева соответствует узловой вершине, их типы можно выбрать не более чем $5^{T}$ способами. Тяжелое ребро $A_{1}$, содержащее фиксированную вершину $v$, можно выбрать не более чем $\Delta$ способами. Место ребра $A_{1}$ в дереве зависимостей можно выбрать не более чем $T$ способами. Далее, каждое следующее ребро в конфигурации гиперграфа соединяется с одним из предыдущих по узловой вершине. Мы сначала поднимаемся вверх до корня, а затем идем вниз согласно нумерации вершин дерева. Каждый раз узловую вершину можно выбрать не более чем $n$ способами. А новое ребро:

- не более чем $\delta$ способами, если узловая вершина - легкая (третий и четвертый тип);

- не более чем $\delta$ способами, если узловая вершина является тяжелой первого или второго типа (мы присоединяем тяжелое ребро);

- не более чем $\delta$ способами, если узловая вершина является тяжелой нулевого типа и мы идем вниз от корня (присоединяем тяжелое ребро);

- не более чем $\Delta$ способами, если узловая вершина является тяжелой нулевого типа и мы идем вверх к корню (присоединяем легкое ребро).

Таким образом, только в последнем случае выбор ребра не оценивается сверху величиной $\delta$. Покажем, однако, что последний вариант является крайне редким.

Утверждение 1. При прямом движении к корню от любой вершины дерева зависимостей второго этапа перекраски узловая вершина нулевого уровня встречается не более одного раза.

Доказательство. Данный факт легко вытекает из построения дерева зависимостей на втором этапе. Если двигаться вниз по дереву, начиная с корня, то, как 
несложно понять, первая встреченная узловая тяжелая вершина будет иметь нулевой или первый тип, это будет первая вершина, присоединяющая старого потомка. Все последующие узловые вершины также будут присоединять старых потомков и будут иметь либо первый, либо второй тип (их перекраска или блокировка происходила еще на первом этапе перекраски).

Подведем промежуточные итоги. При фиксированном выборе структуры дерева, положения в нем ребра, содержащего вершину $v$, и выборе типов узловых вершин все ребра гипердерева можно выбрать не более чем $\Delta(n \delta)^{T-2}(n \Delta)$ способами. Следовательно, из (17) получаем

$$
w_{4}(z) \leqslant \sum_{T=h+1}^{+\infty} \frac{4^{T}}{T} T 5^{T} \Delta(n \delta)^{T-2}(n \Delta)\left(\frac{1}{r}\right)^{(n-1) T} \frac{1}{(n / 2-h-s)^{T-1}} z^{n T} .
$$

При $z=z_{0}=1+1 / n, h+s<n / 4$ и $80 е c<1$ имеем

$$
\begin{gathered}
w_{4}\left(z_{0}\right) \leqslant \sum_{T=h+1}^{+\infty}(20 e)^{T} n^{2 \alpha} c^{T-2} 4^{T-1} \leqslant 1600 e^{2} n^{2 \alpha} \sum_{T=h+1}^{+\infty}(80 e c)^{T-2} \leqslant \\
\leqslant \frac{1600 e^{2} n^{2 \alpha}}{1-80 e c}(80 e c)^{h-1} .
\end{gathered}
$$

Осталось разобрать случай, когда ребра, входящие в $\mathcal{T}$, могут образовывать гиперцикл. Здесь, как и раньше, возможны две ситуации: либо есть поддерево без гиперциклов размера не меньше $q$, либо простой цикл размера не более $2 q$. Второй случай был уже разобран нами в рамках анализа первого этапа перекраски (там мы использовали только изначальную одноцветность или почти одноцветность множества ребер). А анализ большого поддерева полностью повторяет рассуждения для аналогичного случая первого этапа. Для оценки вклада достаточно умножить на $n$ оценку (18) и заменить $h+1$ на $q$. Значит,

$$
w_{5}\left(z_{0}\right) \leqslant \frac{1600 e^{2} n^{2 \alpha+1}}{1-80 e c}(80 e c)^{q-2} .
$$

Перейдем к анализу последнего плохого события.

\section{5. Анализ возникновения большого числа изменений цветов Един-} ственный оставшийся неразобранным случай (плохое событие типа $\mathcal{D}$ ) состоит в том, что некоторое ребро $A \in E$ не было одноцветным или почти одноцветным цвета $\alpha$ в начальной раскраске $\xi$, но стало одноцветным цвета $\alpha$ к концу второго этапа перекраски. Тогда в этом ребре было изначально не менее $s+1$ вершин другого цвета и все они сменили цвет в процессе перекраски. Пусть $v_{1}, \ldots, v_{m_{1}}$ - это все подобные легкие вершины ребра $A$, а $v_{m_{1}+1}, \ldots, v_{m_{1}+m_{2}}$ - тяжелые, где $m_{1}+m_{2} \geqslant s$.

Вершина $v_{i}$ при $i>m_{1}$ является тяжелой, а потому она должна была сменить свой цвет на первом этапе. Обозначим через $B_{i}$ тяжелое ребро, которое повлияло на $v_{i}$. Заметим, что $B_{i}$ обязано быть одноцветным в начальной раскраске $\xi$. Если же $i \leqslant m_{1}$, то вершина $v_{i}$ перекрашивалась на втором этапе, пусть ребро $C_{i}$ повлияло на нее. Тогда $C_{i}$ - легкое ребро, одноцветное в промежуточной раскраске $\zeta$ и почти одноцветное в $\xi$. Тогда в начальной раскраске оно могло иметь ряд тяжелых вершин, не более $s, w_{i}^{1}, \ldots, w_{i}^{x_{i}}, x_{i} \leqslant s$, имевших отличный от цвета $v_{i}$ цвет 
в $\xi$. Обозначим через $D_{i}^{1}, \ldots, D_{i}^{x_{i}}$ одноцветные в $\xi$ тяжелые ребра, повлиявшие на перекраску $w_{i}^{1}, \ldots, w_{i}^{x_{i}}$.

Обозначим через $\mathcal{T}=\left(A, C_{1}, \ldots, C_{m_{1}}, B_{m_{1}+1}, \ldots, B_{m_{1}+m_{2}}, D_{1}^{1}, \ldots, D_{m_{1}}^{x_{m_{1}}}\right)$ получившийся набор ребер. Заметим, что $\mathcal{T}$ - это гипердерево в силу того, что обхват гиперграфа $H$ строго больше пяти. Обозначим вышеописанное событие через $\mathcal{D}(\mathcal{T})$ и оценим его вероятность. При фиксированном выборе цветов ребер $\alpha, \gamma_{1}, \ldots, \gamma_{m_{1}}, \beta_{m_{1}+1}, \ldots, \beta_{m_{1}+m_{2}}, \theta_{1}^{1}, \ldots, \theta_{m_{1}}^{x_{m_{1}}}$, где цвет ребра $A$ - это его цвет в финальной раскраске, а для остальных - доминирующий в $\xi$ цвет, определены изначальные цвета всех вершин гипердерева. Также однозначно определены значения случайных величин $\eta_{w}$ для вершин, лежащих в пересечении ребер, $v_{1}, \ldots, v_{m_{1}+m_{2}}, w_{1}^{1}, \ldots, w_{m_{1}}^{x_{m_{1}}}$. Наконец, для этих вершин должны быть выполнены условия для перекраски, они должны иметь номер не более $h$ в соответствующем множестве тяжелых или легких вершин:

$$
\begin{gathered}
\sigma\left(v_{i},\left(C_{i}\right)_{L}\right) \leqslant h, i \leqslant m_{1} ; \\
\sigma\left(v_{i},\left(B_{i}\right)_{R}\right) \leqslant h, i>m_{1} ; \\
\sigma\left(w_{i}^{j},\left(D_{i}^{j}\right)_{R}\right) \leqslant h .
\end{gathered}
$$

Все указанные множества вершин не пересекаются, а потому события независимы. Стало быть,

$$
\begin{aligned}
\mathrm{P}(\mathcal{D}(\mathcal{T}) \mid \alpha, \ldots, & \left.\theta_{m_{1}}^{x_{m_{1}}}\right) \leqslant\left(\frac{1}{r}\right)^{(n-1)\left(1+m_{1}+m_{2}+x_{1}+\ldots+x_{m_{1}}\right)+1}(r-1)^{-\left(m_{1}+m_{2}+x_{1}+\ldots+x_{m_{1}}\right)_{\times}} \\
& \times\left(\prod_{i=1}^{m_{1}} \frac{h}{\left|\left(C_{i}\right)_{L}\right|}\right)\left(\prod_{i=m_{1}+1}^{m_{1}+m_{2}} \frac{h}{\left|\left(B_{i}\right)_{R}\right|}\right)\left(\prod_{i=1}^{m_{1}} \prod_{j=1}^{x_{i}} \frac{h}{\left|\left(D_{i}^{j}\right)_{R}\right|}\right) \leqslant
\end{aligned}
$$

(т.к. мощности подмножеств не меньше $n / 2$ )

$\leqslant\left(\frac{1}{r}\right)^{(n-1)\left(1+m_{1}+m_{2}+x_{1}+\ldots+x_{m_{1}}\right)+1}(r-1)^{-\left(m_{1}+m_{2}+x_{1}+\ldots+x_{m_{1}}\right)}\left(\frac{2 h}{n}\right)^{m_{1}+m_{2}+x_{1}+\ldots+x_{m_{1}}}$.

Суммируя по всем возможным $r(r-1)^{m_{1}+m_{2}+x_{1}+\ldots+x_{m_{1}}}$ выборам цветов, получаем, что

$$
\mathrm{P}(\mathcal{D}(\mathcal{T})) \leqslant\left(\frac{1}{r}\right)^{(n-1)\left(1+m_{1}+m_{2}+x_{1}+\ldots+x_{m_{1}}\right)}\left(\frac{2 h}{n}\right)^{m_{1}+m_{2}+x_{1}+\ldots+x_{m_{1}}} .
$$

Осталось оценить вклад в локальный полином событий типа $\mathcal{D}(\mathcal{T})$. Пусть $v-$ фиксированная вершина гиперграфа. Если $v$ принадлежит ребру $A$, то:

- само $A$ можно выбрать не более чем $\Delta$ способами;

- ребра $C_{1}, \ldots, C_{m_{1}}$ - не более чем $\left(\begin{array}{c}n \delta \\ m_{1}\end{array}\right)$ способами (т.к. общие вершины должны быть легкими);

- ребра $B_{m_{1}+1}, \ldots, B_{m_{1}+m_{2}}-$ не более чем $\left(\begin{array}{c}n \delta \\ m_{2}\end{array}\right)$ способами (все ребра - тяжелые);

- pебра $D_{1}^{1}, \ldots, D_{m_{1}}^{x_{m_{1}}}$ - не более чем $\prod_{i=1}^{m_{1}}\left(\begin{array}{c}n \delta \\ x_{i}\end{array}\right)$ способами.

Если $v$ принадлежит ребру $C_{j}$, то:

- ребро $C_{j}$ можно выбрать не более чем $\Delta$ способами;

- ребро $A$ - не более чем $n \delta$ способами;

- ребра $C_{1}, \ldots, C_{m_{1}}$, кроме $C_{j},-$ не более чем $\left(\begin{array}{c}n \delta-1 \\ m_{1}-1\end{array}\right)$ способами; 
- ребра $B_{m_{1}+1}, \ldots, B_{m_{1}+m_{2}}$ - не более чем $\left(\begin{array}{c}n \delta \\ m_{2}\end{array}\right)$ способами;

- pебра $D_{1}^{1}, \ldots, D_{m_{1}}^{x_{m_{1}}}$ - не более чем $\prod_{i=1}^{m_{1}}\left(\begin{array}{c}n \delta \\ x_{i}\end{array}\right)$ способами.

Если $v$ принадлежит ребру $B_{j}$, то

- ребро $B_{j}$ можно выбрать не более чем $\delta n$ способами;

- ребро $A$ - не более чем $n \Delta$ способами;

- ребра $C_{1}, \ldots, C_{m_{1}}$ - не более чем $\left(\begin{array}{c}n \delta \\ m_{1}\end{array}\right)$ способами;

- ребра $B_{m_{1}+1}, \ldots, B_{m_{1}+m_{2}}$, кроме $B_{j}$, - не более чем $\left(\begin{array}{c}n \delta-1 \\ m_{2}-1\end{array}\right)$ способами;

- pебра $D_{1}^{1}, \ldots, D_{m_{1}}^{x_{m_{1}}}$ - не более чем $\prod_{i=1}^{m_{1}}\left(\begin{array}{c}n \delta \\ x_{i}\end{array}\right)$ способами.

Наконец, если $v$ принадлежит одному из ребер $D_{i}^{j}$, то

- pебро $D_{i}^{j}$ можно выбрать не более чем $\delta n$ способами;

- ребро $C_{i}$ - не более чем $n \Delta$ способами;

- ребро $A$ - не более чем $n \delta$ способами;

- ребра $C_{1}, \ldots, C_{m_{1}}$, кроме $C_{i}$ - не более чем $\left(\begin{array}{c}n \delta-1 \\ m_{1}-1\end{array}\right)$ способами;

- ребра $B_{m_{1}+1}, \ldots, B_{m_{1}+m_{2}}$ - не более чем $\left(\begin{array}{c}n \delta \\ m_{2}\end{array}\right)$ способами;

- ребра $D_{1}^{1}, \ldots, D_{m_{1}}^{x_{m_{1}}}$, кроме $D_{i}^{j}$, - не более чем $\frac{x_{i}}{n \delta} \prod_{k=1}^{m_{1}}\left(\begin{array}{l}n \delta \\ x_{k}\end{array}\right)$ способами.

Таким образом, получается следующая оценка числа гипердеревьев:

$$
\begin{aligned}
& \left(1+m_{1}+m_{2}+m_{1}\left(x_{1}+\ldots+x_{m_{1}}\right)\right) \Delta\left(\begin{array}{c}
n \delta \\
m_{1}
\end{array}\right)\left(\begin{array}{c}
n \delta \\
m_{2}
\end{array}\right) \prod_{i=1}^{m_{1}}\left(\begin{array}{c}
n \delta \\
x_{i}
\end{array}\right) \leqslant \\
& \leqslant\left(1+m_{1}+m_{2}+s m_{1}^{2}\right) \Delta(n \delta)^{m_{1}+m_{2}+x_{1}+\ldots+x_{m_{1}}} \frac{1}{m_{1} ! m_{2} !} \prod_{i=1}^{m_{1}} \frac{1}{x_{i} !},
\end{aligned}
$$

так как $x_{i} \leqslant s$ при всех $i$. Объединяя данную оценку с (20), мы получаем следующую оценку вклада рассматриваемых событий в локальный полином:

$$
\begin{gathered}
w_{6}\left(z_{0}\right) \leqslant \sum_{m=s+1}^{n} \sum_{m_{1}, m_{2}: m_{1}+m_{2}=m} \sum_{x_{1}, \ldots, x_{m_{1}}=1}^{s}\left(\frac{1}{r}\right)^{(n-1)\left(1+m_{1}+m_{2}+x_{1}+\ldots+x_{m_{1}}\right)} \times \\
\times\left(\frac{2 h}{n}\right)^{m_{1}+m_{2}+x_{1}+\ldots+x_{m_{1}}}\left(1+m_{1}+m_{2}+s m_{1}^{2}\right) \Delta(n \delta)^{m_{1}+m_{2}+x_{1}+\ldots+x_{m_{1}}} \times \\
\times \frac{1}{m_{1} ! m_{2} !}\left(\prod_{i=1}^{m_{1}} \frac{1}{x_{i} !}\right) \cdot z_{0}^{n\left(1+m_{1}+m_{2}+x_{1}+\ldots+x_{m_{1}}\right)} \leqslant
\end{gathered}
$$

(используем условие $(8)$ и $\delta=c r^{n-1}$ )

$$
\begin{gathered}
\leqslant \sum_{m=s+1}^{n} \sum_{m_{1}, m_{2}: m_{1}+m_{2}=m} \sum_{x_{1}, \ldots, x_{m_{1}}=1}^{s} n^{\alpha} \frac{\left(1+m_{1}+m_{2}+s m_{1}^{2}\right)(2 c h)^{m_{1}+m_{2}}}{m_{1} ! m_{2} !}\left(\prod_{i=1}^{m_{1}} \frac{(2 c h)^{x_{i}}}{x_{i} !}\right) \times \\
\times z_{0}^{n\left(1+m_{1}+m_{2}+x_{1}+\ldots+x_{m_{1}}\right)} \leqslant
\end{gathered}
$$

$\left(\right.$ так как $z_{0}=1+1 / n$, то $\left.z_{0}^{n}<e\right)$

$$
\begin{gathered}
\leqslant \sum_{m=s+1}^{n} \sum_{m_{1}, m_{2}: m_{1}+m_{2}=m} \sum_{x_{1}, \ldots, x_{m_{1}}=1}^{s}\left(e n^{\alpha}\right) \times \\
\times \frac{\left(1+m_{1}+m_{2}+s m_{1}^{2}\right)(2 e c h)^{m_{1}+m_{2}}}{m_{1} ! m_{2} !}\left(\prod_{i=1}^{m_{1}} \frac{(2 e c h)^{x_{i}}}{x_{i} !}\right)=
\end{gathered}
$$


$=\sum_{m=s+1}^{n} \sum_{m_{1}, m_{2}: m_{1}+m_{2}=m}\left(e n^{\alpha}\right) \frac{\left(1+m_{1}+m_{2}+s m_{1}^{2}\right)(2 e c h)^{m_{1}+m_{2}}}{m_{1} ! m_{2} !} \prod_{i=1}^{m_{1}}\left(\sum_{x_{i}=0}^{s} \frac{(2 e c h)^{x_{i}}}{x_{i} !}\right) \leqslant$ (оцениваем $\sum_{x_{i}=0}^{s} \frac{(2 e c h)^{x_{i}}}{x_{i} !}$ экспонентой $e^{2 c e h}$ )

$$
\leqslant e n^{\alpha} \sum_{m=s+1}^{n} \sum_{m_{1}, m_{2}: m_{1}+m_{2}=m} \frac{\left(1+m_{1}+m_{2}+s m_{1}^{2}\right)(2 e c h)^{m_{1}+m_{2}}}{m_{1} ! m_{2} !} e^{2 c e h m_{1}} \leqslant
$$

(заметим, что $1+m_{1}+m_{2}+s m_{1}^{2} \leqslant(s+1) m^{2}$ и $m_{1} \leqslant m$ )

$$
\begin{gathered}
\leqslant e n^{\alpha} \sum_{m=s+1}^{n} \frac{(s+1) m^{2}(2 e c h)^{m} e^{2 c h m}}{m !} \sum_{m_{1}, m_{2}: m_{1}+m_{2}=m} \frac{m !}{m_{1} ! m_{2} !}= \\
=e n^{\alpha} \sum_{m=s+1}^{n} \frac{(s+1) m^{2}(4 e c h)^{m} e^{2 c h m}}{m !} \leqslant \\
\leqslant e n^{\alpha+1}\left(4 e^{2} c h\right)^{2} e^{4 c h} \sum_{m=s+1}^{n}\left(\frac{\left(4 e^{2} c h\right) e^{2 c h}}{m}\right)^{m-2},
\end{gathered}
$$

так как $s+1 \leqslant n, m !>(m / e)^{m}$. Если предположить, что $\left(4 e^{2} c h\right) e^{2 c h}<s / 2$, то получается следующее неравенство

$$
w_{6}\left(z_{0}\right) \leqslant e n^{\alpha+3} 2^{2-s} .
$$

4.6. Выбор параметров и завершение доказательства Мы готовы завершить доказательство теоремы. Возьмем в качестве $w(z)$ сумму $\sum_{i=1}^{6} w_{i}(z)$. Для применения Локальной леммы (теорема (4)) нам достаточно показать, что

$$
w\left(z_{0}\right)=w\left(\frac{1}{1-\tau}\right) \leqslant \tau, \quad \text { где } \tau=\frac{1}{n+1} .
$$

Осуществим следующий выбор параметров $h, s, q$ :

$$
h=q=\lceil\ln n\rceil, \quad s=\left\lceil n^{1 / 2}\right\rceil .
$$

Тогда легко видно, что при всех достаточно больших $n$ и $c<1 / 4$ будут выполнены те условия, которые были нам необходимы для получения оценок $w_{i}\left(z_{0}\right)$ :

$$
h+s<n / 4, \quad\left(4 e^{2} c h\right) e^{2 c h}<s / 2 .
$$

В итоге, из оценок (13), (14), (16), (18), (19) и (21) при данном выборе параметров вытекает, что

$$
\begin{gathered}
w\left(z_{0}\right)=\sum_{i=1}^{6} w_{i}\left(z_{0}\right) \leqslant \frac{4 e n^{\alpha}}{1-16 e c}(16 e c)^{h}+\frac{4 e n^{\alpha+1}}{1-16 e c}(16 e c)^{q-1}+2 q e^{4 q} n^{2 q(\alpha+s)} 2^{2 q+1-n}+ \\
\quad+\frac{1600 e^{2} n^{2 \alpha}}{1-80 e c}(80 e c)^{h-1}+\frac{1600 e^{2} n^{2 \alpha+1}}{1-80 e c}(80 e c)^{q-2}+e n^{\alpha+3} 2^{2-s} .
\end{gathered}
$$


Заметим, что при $h=q \sim \ln n$, а $s \sim n^{1 / 2}$ и подходящем выборе (в зависимости от $\alpha$ ) очень маленькой константы $c$

$$
\begin{gathered}
n^{2 \alpha+1}(80 e c)^{\ln n}=o\left(\frac{1}{n}\right), n^{\alpha+3} 2^{-\sqrt{n}}=o\left(\frac{1}{n}\right) \text { и } \\
q e^{4 q} n^{2 q(\alpha+s)} 2^{2 q+1-n}=2^{-n+O\left(\sqrt{n}(\ln n)^{2}\right)}=o\left(\frac{1}{n}\right) .
\end{gathered}
$$

Стало быть, существует такая константа $c=c(\alpha)>0$, что для всех достаточно больших $n$ правая часть $(22)$ будет не превосходить $1 /(n+1)$. Значит, к нашему набору плохих событий применима Локальная лемма, которая утверждает, что с положительной вероятностью ни одно из них не будет выполнено. Следовательно, с положительной вероятностью итоговая случайная раскраска будет правильной для гиперграфа $H$. Теорема 3 доказана.

\section{5. Заключение}

Несмотря на то, что теорема 3 формально выполнена только для гиперграфов с обхватом больше пяти, ее утверждение может быть доказано и для класса простых гиперграфов, что полностью усилит теорему 2 Косточки и Кумбхата из [7]. Единственное место в доказательстве, где использовалось отсутствие циклов длины не более пяти - это анализ плохого события типа $\mathcal{D}$. Здесь потребовалось бы дополнительное рассмотрение ситуаций с короткими циклами (аналогичных тем, что были рассмотрены для других плохих событий), а это заняло бы не одну страницу текста. Однако для простых гиперграфов мы не получаем новых оценок для числа ребер, в связи с чем было решено оставить этот случай без доказательства.

\section{Список литературы}

1. Erdős P., Lovász L., "Problems and results on 3-chromatic hypergraphs and some related questions", Infinite and Finite Sets, Colloquia Mathematica Societatis Janos Bolyai, 10, North Holland, Amsterdam, 1973, 609-627.

2. Райгородский А. М., Шабанов Д. А., “Задача Эрдеша - Хайнала о раскрасках гиперграфов, ее обобщения и смежные проблемы", Успехи математических наук, 66:5 (2011), 109-182.

3. Kostochka A. V., Rődl V., "Constructions of sparse uniform hypergraphs with high chromatic number", Random Structures and Algorithms, 36:1 (2010), 46-56.

4. Kozik J., Shabanov D. A., "Improved algorithms for colorings of simple hypergraphs and applications", arXiv:1409.6921.

5. Kostochka A. V., Mubayi D., Rődl V., Tetali P., "On the chromatic number of set systems", Random Struct. Algor., 19:2 (2001), 87-98.

6. Sauer N., "On the existence of regular $n$-graphs with given girth", J. Comb. Theory, Ser. $B, 9$ (1970), 144-147.

7. Kostochka A. V., Kubmhat M., "Coloring uniform hypergraphs with few edges", Random Struct. Algor., 35:3 (2009), 348-368.

8. Купавский А. Б., Шабанов Д. А., "Раскраски однородных гиперграфов с большим обхватом", Доклады Академии Наук, 443:4 (2012), 422-426.

9. Алон Н., Спенсер Дж., Вероятностный метод, Бином. Лаборатория знаний, М., 2007. 